\title{
A Luminescent Tetrameric Zinc(II) Complex containing the 7-Azaindolate Ligand. Photophysical Properties and Crystal Structure $\dagger$
}

\author{
Chor-Fain Lee, ${ }^{a}$ Kwok-Fai Chin, ${ }^{b}$ Shie-Ming Peng *a and Chi-Ming Che ${ }^{*, b}$ \\ a Department of Chemistry, National Taiwan University, Taipei, Taiwan \\ ${ }^{b}$ Department of Chemistry, The University of Hong Kong, Pokfulam Road, Hong Kong
}

\begin{abstract}
Reaction of $\mathrm{Zn}$ " with 7-azaindole in methanol and in the presence of triethylamine gave a tetrameric zinc(II) complex characterized by $X$-ray crystallography to be $\left[\mathrm{Zn}_{4} \mathrm{O}\left(\mathrm{C}_{7} \mathrm{H}_{5} \mathrm{~N}_{2}\right)_{6}\right]$. The measured intramolecular $\mathrm{Zn} \cdots \mathrm{Zn}$ separations and $\mathrm{Zn}-\mathrm{O}$ distances are 3.147(2)-3.209(2) and 1.903(8)-1.975(8) $\AA$ respectively. At room temperature the cluster displays intense photoluminescence at $448 \mathrm{~nm}$ in the solid state and $425 \mathrm{~nm}$ in acetonitrile. The lifetime and quantum yield of the emission are $0.1 \mu \mathrm{s}$ and 0.17 respectively.
\end{abstract}

Polynuclear $\mathrm{d}^{10}$ metal complexes are of current interest because of their intriguing structural and photoluminescent properties. ${ }^{1-4}$ Despite numerous studies on gold-, copper- and silver(I) systems, ${ }^{2-4}$ there is only one report on luminescent zinc(II) clusters. Vogler and Kunkely ${ }^{4 d}$ first reported the absorption and emission spectra of $\left[\mathrm{Zn}_{4} \mathrm{O}\left(\mathrm{O}_{2} \mathrm{CMe}\right)_{6}\right]$ which is regarded as a molecular model for $\mathrm{ZnO}{ }^{4 d, 5}$ However, the electronic excited state of this complex has a lifetime of $10 \mathrm{~ns}$, which is too short for bimolecular photochemical reactions. Herein is described a new tetrameric zinc(II) cluster with 7-azaindolate as bridging ligand. This complex is of interest because it possesses a long-lived and high-energy electronic excited state in fluid solution at room temperature.

\section{Experimental}

Materials.-The chemicals for synthesis were of analytical grade. Organic solvents for photoluminescent studies were distilled twice prior to use.

Preparation of $\left[\mathrm{Zn}_{4} \mathrm{O}\left(\mathrm{C}_{7} \mathrm{H}_{5} \mathrm{~N}_{2}\right)_{6}\right]$.-A solution of 7-azaindole (1 $H$-pyrrolo[2,3-b]pyridine) $(1.5 \mathrm{mmol})$ in methanol $(20$ $\mathrm{cm}^{3}$ ) was heated to boiling, mixed with a solution of zinc acetate dihydrate $\left(1 \mathrm{mmol}\right.$ in $30 \mathrm{~cm}^{3}$ methanol) and triethylamine (1 $\left.\mathrm{cm}^{3}\right)$ was added. The colourless complex $\left[\mathrm{Zn}_{4} \mathrm{O}\left(\mathrm{C}_{7} \mathrm{H}_{5} \mathrm{~N}_{2}\right)_{6}\right]$. $\mathrm{MeOH}(40 \%$ yield) was obtained after filtration. UV/VIS $\left(\mathrm{CH}_{2} \mathrm{Cl}_{2}\right.$ solution): $296\left(\varepsilon=3.17 \times 10^{4}\right)$ and $325 \mathrm{~nm}(\mathrm{sh})$ $\left(\varepsilon=1.13 \times 10^{4} \mathrm{dm}^{3} \mathrm{~mol}^{-1} \mathrm{~cm}^{-1}\right)$. IR $(\mathrm{KBr}): 3082 \mathrm{w}, 1579 \mathrm{~s}$ $1558 \mathrm{~m}, 1464 \mathrm{~s}, 1423 \mathrm{vs}, 1337 \mathrm{~s}, 1280 \mathrm{vs}, 1263 \mathrm{~m}, 1160 \mathrm{vs}, 1118 \mathrm{w}$, $1067 \mathrm{w}, 1053 \mathrm{w}, 939 \mathrm{w}, 913 \mathrm{~m}, 797 \mathrm{~m}, 789 \mathrm{~m}, 767 \mathrm{~m}, 732 \mathrm{~m}, 638 \mathrm{w}$ $611 \mathrm{w}, 577 \mathrm{w}, 520 \mathrm{w}$ and $468 \mathrm{w} \mathrm{cm}^{-1}$ (Found: $\mathrm{C}, 49.85 ; \mathrm{H}, 2.80 ; \mathrm{N}$ 16.10. Calc. for $\mathrm{C}_{42} \mathrm{H}_{30} \mathrm{~N}_{12} \mathrm{OZn}_{4} \cdot \mathrm{CH}_{4} \mathrm{O}: \mathrm{C}, 50.95 ; \mathrm{H}, 3.35 ; \mathrm{N}$, $16.60 \%$ ).

Structure Determination.- The crystals of $\left[\mathrm{Zn}_{4} \mathrm{O}\left(\mathrm{C}_{7} \mathrm{H}_{5}\right.\right.$ $\left.\left.\mathrm{N}_{2}\right)_{6}\right] \cdot \mathrm{CH}_{2} \mathrm{Cl}_{2} \cdot \mathrm{H}_{2} \mathrm{O}$ used were obtained by slow diffusion of diethyl ether into a methylene chloride solution of $\left[\mathrm{Zn}_{4} \mathrm{O}\left(\mathrm{C}_{7} \mathrm{H}_{5} \mathrm{~N}_{2}\right)_{6}\right]$. To prevent loss of solvent the crystals were sealed in a layer of epoxy resin. The data were collected on an Enraf-Nonius CAD4 four-circle diffractometer. Unit-cell constants were obtained by a least-squares fit to the setting angles of 25 strong reflections. Intensity data were collected

$\dagger$ Supplementary data available: see Instructions for Authors, J. Chem Soc., Dalton Trans., 1993, Issue 1, pp. xxiii-xxviii.
Table 1 Crystallographic parameters

$\begin{array}{ll}\text { Formula } & \mathrm{C}_{42} \mathrm{H}_{30} \mathrm{~N}_{12} \mathrm{OZn}_{4} \cdot \mathrm{CH}_{2} \mathrm{Cl}_{2} \cdot \mathrm{H}_{2} \\ M & 1072.19 \\ \text { Colour } & \text { Colourless } \\ \text { Crystal system } & \text { Triclinic } \\ \text { Space group } & P \overline{1} \\ a / \AA & 10.263(1) \\ b / \AA & 10.447(1) \\ c / \AA & 20.817(3) \\ \alpha / /^{\circ} & 95.09(1) \\ \beta / /^{\circ} & 94.22(1) \\ \gamma / /^{\circ} & 96.26(1) \\ U / \AA^{3} & 2201.9(5) \\ Z & 2 \\ \text { Crystal dimensions/mm } & 0.08 \times 0.12 \times 0.17 \\ F(000) & 1092 \\ D \text { c/g cm } & -3 \\ \text { Scan parameter } & 1.617 \\ \text { Scan speed/ min } & \\ \text { Maximum, minimum transmission } & 0.8+0.35 \tan \theta \\ 2 \theta \text { limit }{ }^{\circ} & 16.48 / 7 \text { to } 16.48 / 2 \\ h k l \text { ranges } & 1.00,0.87 \\ \text { Reflections collected } & 45 \\ \text { Reflections observed }[I>2 \sigma(I)] & -11 \text { to } 10,0-11,-22 \text { to } 22 \\ \text { No. of variables } & 5727 \\ \text { Maximum peak in difference map/ } & 2907 \\ \text { e } \AA^{-3} & 558 \\ \text { Weighting scheme, } w & 0.640 \\ R & \\ R^{\prime} & {\left[\sigma^{2}(F)+0.0001 F^{2}\right]^{-1}} \\ & 0.060 \\ & 0.054\end{array}$

using graphite-monochromated Mo-K $\alpha$ radiation $(\lambda=0.7093$ $\AA)$ ). $\mathrm{\psi} \psi$-scan absorption correction was applied. The structure was solved by the Patterson method. Final refinement was carried out with anisotropic thermal parameters for all nonhydrogen atoms. Hydrogen atoms were included at idealized positions with a fixed isotropic thermal parameter $U_{\mathrm{H}}=U_{\mathrm{C}}+$ $0.01 \AA^{2}$. Crystallographic programs used were those of NRCCSDP ${ }^{6}$ installed on a Micro Vax III computer. A summary of the crystal data is given in Table 1 . Table 2 lists the atomic coordinates of non-hydrogen atoms and selected bond distances and angles are given in Table 3.

Additional material available from the Cambridge Crystallographic Data Centre comprises $\mathrm{H}$-atom coordinates, thermal parameters and remaining bond lengths and angles. 
Table 2 Non-hydrogen atom coordinates for $\left[\mathrm{Zn}_{4} \mathrm{O}\left(\mathrm{C}_{7} \mathrm{H}_{5} \mathrm{~N}_{2}\right)_{6}\right] \cdot \mathrm{CH}_{2} \mathrm{Cl}_{2} \cdot \mathrm{H}_{2} \mathrm{O}$ with estimated standard deviations (e.s.d.s) in parentheses

\begin{tabular}{|c|c|c|c|c|c|c|c|}
\hline Atom & $x$ & $y$ & $z$ & Atom & $x$ & $y$ & $z$ \\
\hline $\mathrm{Zn}(1)$ & $0.10972(16)$ & $0.61186(15)$ & $0.23846(8)$ & $N(1 D)$ & $-0.1803(10)$ & $0.8999(10)$ & $0.3182(5)$ \\
\hline $\operatorname{Zn}(2)$ & $-0.12590(15)$ & $0.72397(15)$ & $0.30707(8)$ & $N(2 D)$ & $-0.0398(10)$ & $1.0073(9)$ & $0.2482(5)$ \\
\hline $\mathrm{Zn}(3)$ & $-0.01954(15)$ & $0.85128(15)$ & $0.18424(8)$ & C(1D) & $-0.2590(14)$ & $0.9422(13)$ & $0.3619(8)$ \\
\hline $\mathrm{Zn}(4)$ & $-0.17546(17)$ & $0.57097(16)$ & $0.16766(9)$ & $C(2 D)$ & $-0.2531(15)$ & $1.0764(15)$ & $0.3718(8)$ \\
\hline$O(1)$ & $-0.0541(7)$ & $0.6904(7)$ & $0.2264(4)$ & $C(3 D)$ & $-0.1647(14)$ & $1.1175(12)$ & $0.3276(7)$ \\
\hline$N(1 A)$ & $0.1247(10)$ & $0.5545(11)$ & $0.3256(5)$ & $C(4 D)$ & $-0.1126(16)$ & $1.2308(15)$ & $0.3100(8)$ \\
\hline $\mathrm{N}(2 \mathrm{~A})$ & $0.0065(10)$ & $0.7095(11)$ & $0.3813(5)$ & $C(5 D)$ & $-0.0312(16)$ & $1.2379(13)$ & $0.2631(8)$ \\
\hline$C(1 A)$ & $0.2080(14)$ & $0.4639(14)$ & $0.3479(8)$ & $C(6 D)$ & $0.0074(14)$ & $1.1264(13)$ & $0.2322(7)$ \\
\hline $\mathrm{C}(2 \mathrm{~A})$ & $0.2225(16)$ & $0.4705(18)$ & $0.4147(8)$ & $C(7 D)$ & $-0.1234(12)$ & $1.0050(12)$ & $0.2937(6)$ \\
\hline$C(3 A)$ & $0.1468(14)$ & $0.5735(16)$ & $0.4378(7)$ & $\mathrm{N}(1 \mathrm{E})$ & $-0.2871(11)$ & $0.4528(10)$ & $0.2192(6)$ \\
\hline$C(4 A)$ & $0.1235(18)$ & $0.6259(19)$ & $0.4939(8)$ & $N(2 E)$ & $-0.2712(10)$ & $0.5821(10)$ & $0.3224(6)$ \\
\hline$C(5 A)$ & $0.0440(18)$ & $0.7257(19)$ & $0.4972(8)$ & $C(1 E)$ & $-0.3491(17)$ & $0.3350(15)$ & $0.1895(9)$ \\
\hline$C(6 A)$ & $-0.0127(14)$ & $0.7623(16)$ & $0.4419(7)$ & $\mathrm{C}(2 \mathrm{E})$ & $-0.4362(19)$ & $0.2685(18)$ & $0.2291(11)$ \\
\hline$C(7 A)$ & $0.0896(12)$ & $0.6145(13)$ & $0.3794(6)$ & $C(3 E)$ & $-0.4136(15)$ & $0.3848(16)$ & $0.3031(9)$ \\
\hline $\mathrm{N}(1 \mathrm{~B})$ & $0.1609(10)$ & $0.8698(9)$ & $0.1572(5)$ & $\mathrm{C}(4 \mathrm{E})$ & $-0.454(3)$ & $0.392(3)$ & $0.3570(16)$ \\
\hline $\mathrm{N}(2 \mathrm{~B})$ & $0.2666(10)$ & $0.7450(10)$ & $0.2301(6)$ & $C\left(4 E^{\prime}\right)$ & $-0.469(4)$ & $0.278(4)$ & $0.273(3)$ \\
\hline $\mathrm{C}(1 \mathrm{~B})$ & $0.2104(13)$ & $0.9645(13)$ & $0.1207(6)$ & $C(5 E)$ & $-0.4120(17)$ & $0.4800(17)$ & $0.3918(11)$ \\
\hline $\mathrm{C}(2 \mathrm{~B})$ & $0.3443(14)$ & $0.9915(14)$ & $0.1284(7)$ & $\mathrm{C}(6 \mathrm{E})$ & $-0.3231(16)$ & $0.5823(17)$ & $0.3812(8)$ \\
\hline$C(3 B)$ & $0.3871(13)$ & $0.9091(13)$ & $0.1750(6)$ & $C(7 E)$ & $-0.3187(12)$ & $0.4787(12)$ & $0.2787(7)$ \\
\hline$C(4 B)$ & $0.5027(14)$ & $0.8828(15)$ & $0.2053(8)$ & $\mathrm{N}(1 \mathrm{~F})$ & $-0.1619(10)$ & $0.8628(10)$ & $0.1153(5)$ \\
\hline $\mathrm{C}(5 \mathrm{~B})$ & $0.5023(14)$ & $0.7889(16)$ & $0.2454(8)$ & $\mathrm{N}(2 \mathrm{~F})$ & $-0.3022(10)$ & $0.6639(10)$ & $0.1180(5)$ \\
\hline $\mathrm{C}(6 \mathrm{~B})$ & $0.3898(13)$ & $0.7199(14)$ & $0.2589(7)$ & $C(1 F)$ & $-0.1706(14)$ & $0.9735(13)$ & $0.0857(7)$ \\
\hline$C(7 B)$ & $0.2688(12)$ & $0.8367(11)$ & $0.1905(6)$ & $\mathrm{C}(2 \mathrm{~F})$ & $-0.2920(18)$ & $0.9731(16)$ & $0.0461(7)$ \\
\hline $\mathrm{N}(1 \mathrm{C})$ & $0.1189(11)$ & $0.4791(11)$ & $0.1679(5)$ & $C(3 F)$ & $-0.3590(14)$ & $0.8439(16)$ & $0.0543(7)$ \\
\hline $\mathrm{N}(2 \mathrm{C})$ & $-0.0842(12)$ & $0.4703(11)$ & $0.1066(6)$ & $\mathrm{C}(4 \mathrm{~F})$ & $-0.4748(18)$ & $0.7698(22)$ & $0.0357(9)$ \\
\hline$C(1 C)$ & $0.2314(16)$ & $0.4208(14)$ & $0.1602(7)$ & $C(5 F)$ & $-0.5073(19)$ & $0.6613(20)$ & $0.0556(10)$ \\
\hline $\mathrm{C}(2 \mathrm{C})$ & $0.2317(17)$ & $0.3349(16)$ & $0.1027(9)$ & $C(6 F)$ & $-0.4244(15)$ & $0.6064(15)$ & $0.0941(9)$ \\
\hline$C(3 C)$ & $0.0936(17)$ & $0.3453(15)$ & $0.0733(7)$ & $C(7 F)$ & $-0.2727(12)$ & $0.7826(13)$ & $0.0966(6)$ \\
\hline$C(4 C)$ & $0.0285(23)$ & $0.3004(19)$ & $0.0229(11)$ & C & $0.793(4)$ & $0.927(4)$ & $0.5832(20)$ \\
\hline$C(5 C)$ & $-0.0920(19)$ & $0.3278(17)$ & $0.0097(9)$ & $\mathrm{Cl}(1)$ & $0.6159(18)$ & $0.8566(19)$ & $0.5380(9)$ \\
\hline$C(6 C)$ & $-0.1430(17)$ & $0.4161(15)$ & $0.0504(9)$ & $\mathrm{Cl}(2)$ & $0.7181(22)$ & $0.9722(24)$ & $0.6414(11)$ \\
\hline$C(7 C)$ & $0.0355(15)$ & $0.4347(11)$ & $0.1167(6)$ & $\mathrm{O}(2)$ & $0.4580(25)$ & $0.7790(25)$ & $0.4730(12)$ \\
\hline
\end{tabular}

Table 3 Selected bond distances $(\AA)$ and angles $\left({ }^{\circ}\right)$ of $\left[\mathrm{Zn}_{4} \mathrm{O}\left(\mathrm{C}_{7} \mathrm{H}_{5} \mathrm{~N}_{2}\right)_{6}\right] \cdot \mathrm{CH}_{2} \mathrm{Cl}_{2} \mathrm{H}_{2} \mathrm{O}$

\begin{tabular}{|c|c|c|c|c|c|c|c|}
\hline $\operatorname{Zn}(1) \cdots \operatorname{Zn}(2)$ & $3.176(2)$ & $\mathrm{Zn}(1)-\mathrm{N}(2 \mathrm{~B})$ & $2.04(1)$ & $\mathrm{Zn}(2)-\mathrm{N}(1 \mathrm{D})$ & $1.98(1)$ & $\mathrm{Zn}(3)-\mathrm{N}(2 \mathrm{D})$ & $2.05(1)$ \\
\hline $\operatorname{Zn}(1) \cdots \operatorname{Zn}(3)$ & $3.209(2)$ & $\mathrm{Zn}(2) \cdots \mathrm{Zn}(3)$ & $3.193(2)$ & $\mathrm{Zn}(2)-\mathrm{N}(2 \mathrm{E})$ & $2.05(1)$ & $\operatorname{Zn}(3)-N(1 F)$ & $1.99(1)$ \\
\hline $\operatorname{Zn}(1) \cdots \operatorname{Zn}(4)$ & $3.147(2)$ & $\operatorname{Zn}(2) \cdots \operatorname{Zn}(4)$ & $3.165(2)$ & $\operatorname{Zn}(3) \cdots \operatorname{Zn}(4)$ & $3.158(2)$ & $\mathrm{Zn}(4)-\mathrm{O}(1)$ & $1.941(7)$ \\
\hline $\mathrm{Zn}(1)-\mathrm{O}(1)$ & $1.959(7)$ & $\mathrm{Zn}(2)-\mathrm{O}(1)$ & $1.903(8)$ & $\mathrm{Zn}(3)-\mathrm{O}(1)$ & $1.975(8)$ & $\mathrm{Zn}(4)-\mathrm{N}(1 \mathrm{E})$ & $2.02(1)$ \\
\hline $\mathrm{Zn}(1)-\mathrm{N}(1 \mathrm{~A})$ & $1.96(1)$ & $\mathrm{Zn}(2)-\mathrm{N}(2 \mathrm{~A})$ & $2.01(1)$ & $\mathrm{Zn}(3)-\mathrm{N}(1 \mathrm{~B})$ & $1.97(1)$ & $\mathrm{Zn}(4)-\mathrm{N}(2 \mathrm{~F})$ & $1.99(1)$ \\
\hline $\mathrm{Zn}(2)-\mathrm{Zn}(1)-\mathrm{Zn}(3)$ & $60.01(5)$ & $\mathrm{Zn}(1)-\mathrm{Zn}(2)-\mathrm{N}(2 \mathrm{E})$ & $112.6(3)$ & $\mathrm{Zn}(1)-\mathrm{Zn}(3)-\mathrm{N}(2 \mathrm{D})$ & $118.4(3)$ & $\mathrm{Zn}(1)-\mathrm{Zn}(4)-\mathrm{O}(1)$ & $36.4(2)$ \\
\hline $\mathrm{Zn}(2)-\mathrm{Zn}(1)-\mathrm{Zn}(4)$ & $60.08(5)$ & $\operatorname{Zn}(3)-\operatorname{Zn}(2)-\operatorname{Zn}(4)$ & $59.56(5)$ & $\mathrm{Zn}(1)-\mathrm{Zn}(3)-\mathrm{N}(1 \mathrm{~F})$ & $132.8(3)$ & $\mathrm{Zn}(1)-\mathrm{Zn}(4)-\mathrm{N}(1 \mathrm{E})$ & $106.8(3)$ \\
\hline $\mathrm{Zn}(2)-\mathrm{Zn}(1)-\mathrm{O}(1)$ & $34.1(2)$ & $\mathrm{Zn}(3)-\mathrm{Zn}(2)-\mathrm{O}(1)$ & $35.3(2)$ & $\operatorname{Zn}(2)-\operatorname{Zn}(3)-\operatorname{Zn}(4)$ & $59.78(5)$ & $\mathrm{Zn}(1)-\operatorname{Zn}(4)-\mathrm{N}(2 \mathrm{~F})$ & $141.3(3)$ \\
\hline $\mathrm{Zn}(2)-\mathrm{Zn}(1)-\mathrm{N}(1 \mathrm{~A})$ & $75.7(3)$ & $\mathrm{Zn}(3)-\mathrm{Zn}(2)-\mathrm{N}(2 \mathrm{~A})$ & $117.2(3)$ & $\mathrm{Zn}(2)-\mathrm{Zn}(3)-\mathrm{O}(1)$ & $33.8(2)$ & $\operatorname{Zn}(2)-\operatorname{Zn}(4)-\operatorname{Zn}(3)$ & $60.66(5)$ \\
\hline $\mathrm{Zn}(2)-\mathrm{Zn}(1)-\mathrm{N}(2 \mathrm{~B})$ & $114.7(3)$ & $\operatorname{Zn}(3)-\operatorname{Zn}(2)-N(1 D)$ & $77.5(3)$ & $\mathrm{Zn}(2)-\mathrm{Zn}(3)-\mathrm{N}(1 \mathrm{~B})$ & $128.7(3)$ & $\mathrm{Zn}(2)-\mathrm{Zn}(4)-\mathrm{O}(1)$ & $34.2(2)$ \\
\hline $\mathrm{Zn}(3)-\mathrm{Zn}(1)-\mathrm{Zn}(4)$ & $59.58(5)$ & $\mathrm{Zn}(3)-\mathrm{Zn}(2)-\mathrm{N}(2 \mathrm{E})$ & $136.0(3)$ & $\mathrm{Zn}(2)-\mathrm{Zn}(3)-\mathrm{N}(2 \mathrm{D})$ & $76.2(3)$ & $\mathrm{Zn}(2)-\mathrm{Zn}(4)-\mathrm{N}(1 \mathrm{E})$ & $79.2(3)$ \\
\hline $\mathrm{Zn}(3)-\mathrm{Zn}(1)-\mathrm{O}(1)$ & $35.5(2)$ & $\mathrm{Zn}(4)-\mathrm{Zn}(2)-\mathrm{O}(1)$ & $35.0(2)$ & $\mathrm{Zn}(2)-\mathrm{Zn}(3)-\mathrm{N}(1 \mathrm{~F})$ & $112.8(3)$ & $\mathrm{Zn}(2)-\mathrm{Zn}(4)-\mathrm{N}(2 \mathrm{~F})$ & $105.9(3)$ \\
\hline $\operatorname{Zn}(3)-\operatorname{Zn}(1)-N(1 A)$ & $131.3(3)$ & $\mathrm{Zn}(4)-\mathrm{Zn}(2)-\mathrm{N}(2 \mathrm{~A})$ & $131.1(3)$ & $\mathrm{Zn}(4)-\mathrm{Zn}(3)-\mathrm{O}(1)$ & $35.9(2)$ & $\mathrm{Zn}(3)-\mathrm{Zn}(4)-\mathrm{O}(1)$ & $36.6(2)$ \\
\hline $\mathrm{Zn}(3)-\mathrm{Zn}(1)-\mathrm{N}(2 \mathrm{~B})$ & $75.9(3)$ & $\mathrm{Zn}(4)-\mathrm{Zn}(2)-\mathrm{N}(1 \mathrm{D})$ & $117.9(3)$ & $\mathrm{Zn}(4)-\mathrm{Zn}(3)-\mathrm{N}(1 \mathrm{~B})$ & $117.5(3)$ & $\mathrm{Zn}(3)-\mathrm{Zn}(4)-\mathrm{N}(1 \mathrm{E})$ & $139.1(3)$ \\
\hline $\mathrm{Zn}(4)-\mathrm{Zn}(1)-\mathrm{O}(1)$ & $36.0(2)$ & $\mathrm{Zn}(4)-\operatorname{Zn}(2)-\mathrm{N}(2 \mathrm{E})$ & $79.2(4)$ & $\mathrm{Zn}(4)-\mathrm{Zn}(3)-\mathrm{N}(2 \mathrm{D})$ & $129.7(3)$ & $\operatorname{Zn}(3)-\operatorname{Zn}(4)-N(2 F)$ & $80.4(3)$ \\
\hline $\mathrm{Zn}(4)-\mathrm{Zn}(1)-\mathrm{N}(1 \mathrm{~A})$ & $115.5(3)$ & $\mathrm{O}(1)-\mathrm{Zn}(2)-\mathrm{N}(2 \mathrm{~A})$ & $111.0(4)$ & $\operatorname{Zn}(4)-\operatorname{Zn}(3)-N(1 F)$ & $76.6(3)$ & $O(1)-Z n(4)-N(1 E)$ & $109.5(4)$ \\
\hline $\mathrm{Zn}(4)-\mathrm{Zn}(1)-\mathrm{N}(2 \mathrm{~B})$ & 131.1(3) & $\mathrm{O}(1)-\mathrm{Zn}(2)-\mathrm{N}(1 \mathrm{D})$ & $112.1(4)$ & $\mathrm{O}(1)-\mathrm{Zn}(3)-\mathrm{N}(1 \mathrm{~B})$ & $110.5(4)$ & $\mathrm{O}(1)-\mathrm{Zn}(4)-\mathrm{N}(2 \mathrm{~F})$ & $111.3(4)$ \\
\hline $\mathrm{O}(1)-\mathrm{Zn}(1)-\mathrm{N}(1 \mathrm{~A})$ & $109.0(4)$ & $\mathrm{O}(1)-\mathrm{Zn}(2)-\mathrm{N}(2 \mathrm{E})$ & $112.1(4)$ & $\mathrm{O}(1)-\mathrm{Zn}(3)-\mathrm{N}(2 \mathrm{D})$ & $109.5(4)$ & $\mathrm{N}(1 \mathrm{E})-\mathrm{Zn}(4)-\mathrm{N}(2 \mathrm{~F})$ & $105.3(5)$ \\
\hline $\mathrm{O}(1)-\mathrm{Zn}(1)-\mathrm{N}(2 \mathrm{~B})$ & $109.9(4)$ & $N(2 A)-Z n(2)-N(1 D)$ & $107.1(4)$ & $\mathrm{O}(1)-\mathrm{Zn}(3)-\mathrm{N}(1 \mathrm{~F})$ & $110.4(4)$ & $\mathrm{Zn}(1)-\mathrm{O}(1)-\mathrm{Zn}(2)$ & $110.6(4)$ \\
\hline $\mathrm{N}(1 \mathrm{~A})-\mathrm{Zn}(1)-\mathrm{N}(2 \mathrm{~B})$ & $108.5(4)$ & $\mathrm{N}(2 \mathrm{~A})-\mathrm{Zn}(2)-\mathrm{N}(2 \mathrm{E})$ & $100.8(4)$ & $\mathrm{N}(1 \mathrm{~B})-\mathrm{Zn}(3)-\mathrm{N}(2 \mathrm{D})$ & $108.4(4)$ & $\mathrm{Zn}(1)-\mathrm{O}(1)-\mathrm{Zn}(3)$ & $109.3(4)$ \\
\hline $\operatorname{Zn}(1)-\operatorname{Zn}(2)-\operatorname{Zn}(3)$ & $60.50(5)$ & $N(1 D)-Z n(2)-N(2 E)$ & $113.1(4)$ & $\mathrm{N}(1 \mathrm{~B})-\mathrm{Zn}(3)-\mathrm{N}(1 \mathrm{~F})$ & $115.7(4)$ & $\mathrm{Zn}(1)-\mathrm{O}(1)-\mathrm{Zn}(4)$ & $107.6(3)$ \\
\hline $\mathrm{Zn}(1)-\mathrm{Zn}(2)-\mathrm{Zn}(4)$ & $59.50(5)$ & $\operatorname{Zn}(1)-\operatorname{Zn}(3)-\operatorname{Zn}(2)$ & $59.49(5)$ & $\mathrm{N}(2 \mathrm{D})-\mathrm{Zn}(3)-\mathrm{N}(1 \mathrm{~F})$ & $101.7(4)$ & $\mathrm{Zn}(2)-\mathrm{O}(1)-\mathrm{Zn}(3)$ & $110.9(3)$ \\
\hline $\mathrm{Zn}(1)-\mathrm{Zn}(2)-\mathrm{O}(1)$ & $35.3(2)$ & $\operatorname{Zn}(1)-\operatorname{Zn}(3)-\operatorname{Zn}(4)$ & $59.23(5)$ & $\operatorname{Zn}(1)-\operatorname{Zn}(4)-\operatorname{Zn}(2)$ & $60.42(5)$ & $\mathrm{Zn}(2)-\mathrm{O}(1)-\mathrm{Zn}(4)$ & $110.9(4)$ \\
\hline $\operatorname{Zn}(1)-\operatorname{Zn}(2)-\mathrm{N}(2 \mathrm{~A})$ & $76.6(3)$ & $\mathrm{Zn}(1)-\mathrm{Zn}(3)-\mathrm{O}(1)$ & $35.2(2)$ & $\operatorname{Zn}(1)-\operatorname{Zn}(4)-\operatorname{Zn}(3)$ & $61.18(5)$ & $\mathrm{Zn}(3)-\mathrm{O}(1)-\mathrm{Zn}(4)$ & $107.5(3)$ \\
\hline $\operatorname{Zn}(1)-\operatorname{Zn}(2)-N(1 D)$ & $132.4(3)$ & $\operatorname{Zn}(1)-\operatorname{Zn}(3)-N(1 B)$ & $75.6(3)$ & & & & \\
\hline
\end{tabular}

Physical Measurements.-Emission spectra were measured on a Spex-Fluorolog spectrofluorimeter. Lifetime measurements were conducted with a Quanta-Ray DCR-3 Nd-YAG pulsed laser system. The luminescence quantum yield was determined according to the method of Demas and Crosby. ${ }^{7}$ Sample solutions for photochemical experiments were degassed by at least four successive freeze-pump-thaw cycles.

\section{Results and Discussion}

Tetrameric metal complexes having a $\mathrm{M}_{4} \mathrm{O}(\mathrm{M}=$ metal atom) core are not uncommon. It has been reported that the reactions of 7-azaindolate $\left(\mathrm{C}_{7} \mathrm{H}_{5} \mathrm{~N}_{2}^{-}\right)$with divalent metal ions such as $\mathrm{Co}^{\text {II }}$ and $\mathrm{Cu}^{\text {II }}$ would give complexes of the kind $\left[\mathrm{M}_{4} \mathrm{O}\right.$ $\left.\left(\mathrm{C}_{7} \mathrm{H}_{5} \mathrm{~N}_{2}\right)_{6}\right] .{ }^{8}$ In this work, the zinc(II) cluster was prepared by a similar method. It is an air-stable solid which is 


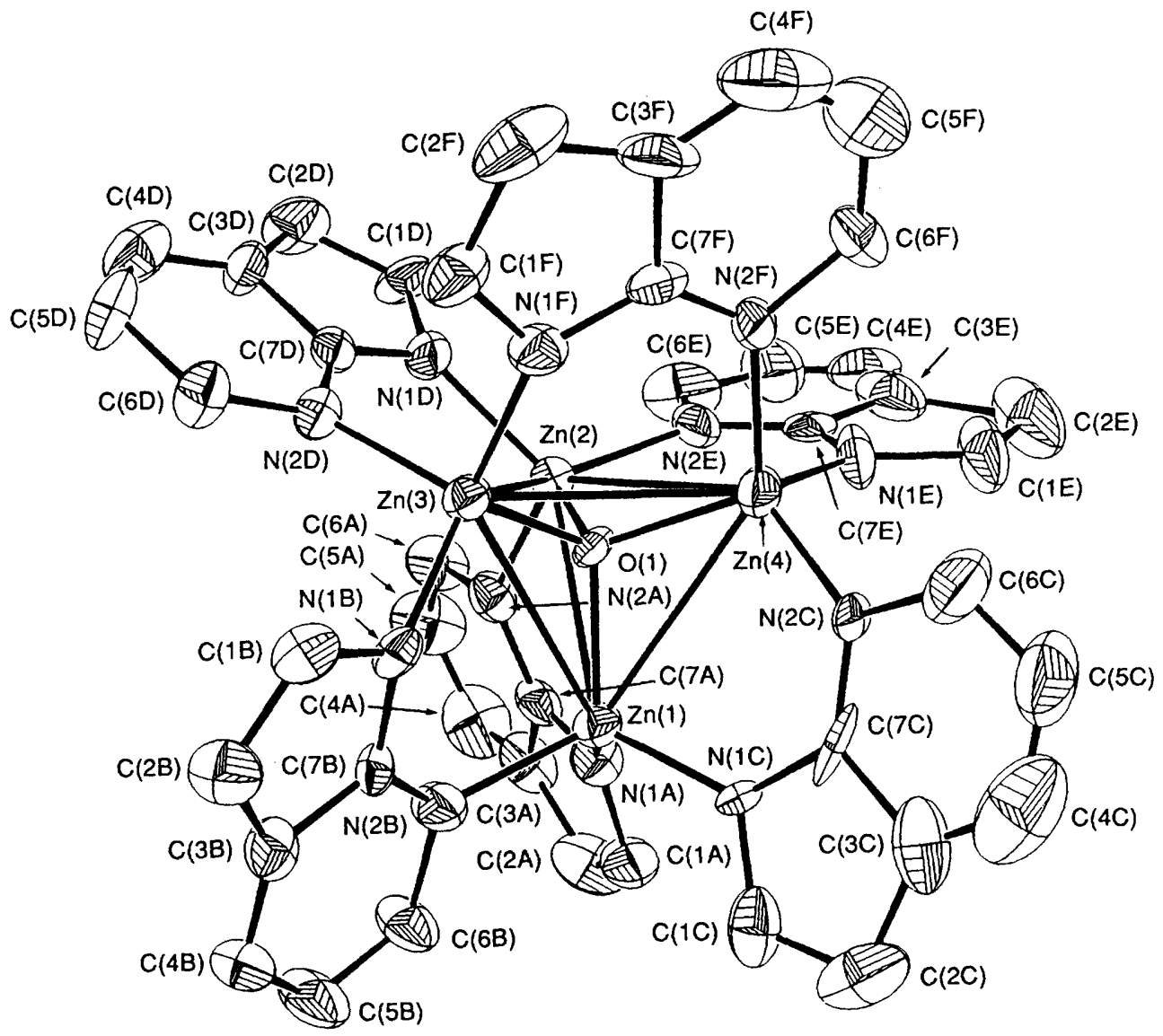

Fig. 1 A perspective view of $\left[\mathrm{Zn}_{4} \mathrm{O}\left(\mathrm{C}_{7} \mathrm{H}_{5} \mathrm{~N}_{2}\right)_{6}\right]$ showing the atom numbering

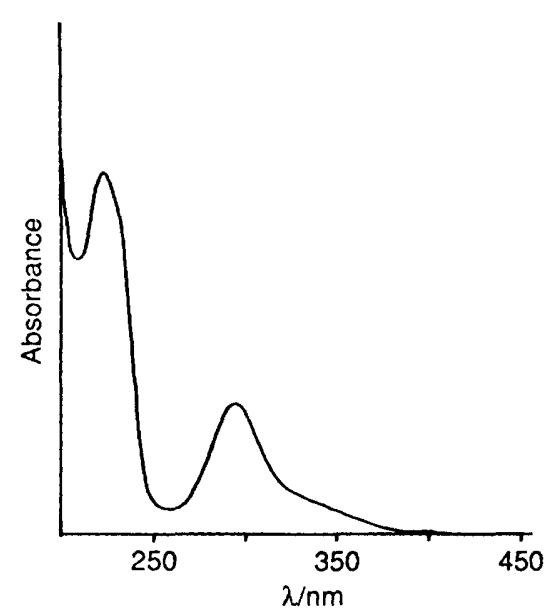

Fig. 2 The UV/VIS absorption spectrum of $\left[\mathrm{Zn}_{4} \mathrm{O}\left(\mathrm{C}_{7} \mathrm{H}_{5} \mathrm{~N}_{2}\right)_{6}\right]$ in acetonitrile

electrochemically inactive in acetonitrile. Although stable in acetonitrile, it undergoes degradation in dichloromethane or chloroform upon exposure to UV light. Fig. 1 shows a perspective view of the molecule with atom numbering. The complex is isostructural to $\left[\mathrm{Zn}_{4} \mathrm{O}\left(\mathrm{O}_{2} \mathrm{CMe}\right)_{6}\right]^{9}$ and $\left[\mathrm{Co}_{4} \mathrm{O}\right.$ $\left.\left(\mathrm{C}_{7} \mathrm{H}_{5} \mathrm{~N}_{2}\right)_{6}\right]^{8}$ in that the central oxygen atom is tetrahedrally surrounded by four metal atoms. If the $\mathrm{Zn} \cdots \mathrm{Zn}$ contacts are ignored, the local stereochemistry of the $\mathrm{ZnN}_{3} \mathrm{O}$ chromophore is tetrahedral with the 7-azaindolate ion serving as a bridging ligand. The measured intramolecular $\mathrm{Zn} \cdots \mathrm{Zn}$ separations of $3.147(2)-3.209(2) \AA$ and $\mathrm{Zn}-\mathrm{O}$ distances of $1.903(8)-1.975(8) \AA$ are close to the values reported for $\left[\mathrm{Zn}_{4} \mathrm{O}\left(\mathrm{O}_{2} \mathrm{CMe}\right)_{6}\right](\mathrm{Zn}-\mathrm{O}$ $1.97, \mathrm{Zn} \cdots \operatorname{Zn} 3.20 \AA){ }^{9}$
Fig. 2 shows the UV/VIS absorption spectrum of the complex in acetonitrile at room temperature. It features intense bands centred at 223 and at $288-294 \mathrm{~nm}$ and a broad shoulder at around $330 \mathrm{~nm}$. The bands at $288-294 \mathrm{~nm}$, which are absent in the spectrum of $\left[\mathrm{Zn}_{4} \mathrm{O}\left(\mathrm{O}_{2} \mathrm{CMe}\right)_{6}\right]^{4 d}$ but present for the free 7 -azaindole ligand, are assigned to the spin-allowed intraligand $\pi \longrightarrow \pi^{*}$ transitions. The nature of the band at $223 \mathrm{~nm}$ is difficult to acertain, since a similar band at $219 \mathrm{~nm}$ is also present for the free ligand. For a tetrameric zinc(II) system of the type $\mathrm{Zn}_{4} \mathrm{O}(\mathrm{L})_{6}$ where $\mathrm{L}$ is a bridging ligand the highest occupied molecular orbital (HOMO) is likely to be a hybrid of the $p_{r}$ orbitals of the central oxygen atom and ligand $L$, whereas the lowest unoccupied molecular orbital (LUMO) $4 S_{\sigma}$ comes from the symmetric combination of the four zinc(II) $4 \mathrm{~s}$ orbitals. ${ }^{4 d}$ Thus, other than the possible intraligand $\pi \longrightarrow \pi^{*}$ transition of $\mathrm{L}$, the ligand-to-metal charge-transfer (1.m.c.t.) transition $\mathrm{p}_{n}\left(\mathrm{O}^{2-}, \mathrm{L}\right) \longrightarrow 4 \mathrm{~S}_{\sigma}$ would also exist. For the $\left[\mathrm{Zn}_{4} \mathrm{O}\right.$ $\left.\left(\mathrm{O}_{2} \mathrm{CMe}\right)_{6}\right]$ system the l.m.c.t. band occurs at around $216 \mathrm{~nm}$. Because $p_{\pi^{*}}$ of 7-azaindolate ion is lower in energy than that of $\mathrm{MeCO}_{2}$ and that the $4 \mathrm{~s}$ orbital may also mix with the $\pi^{*}$ of $\mathrm{L}$, the analogous 1.m.c.t. band of $\left[\mathrm{Zn}_{4} \mathrm{O}\left(\mathrm{C}_{7} \mathrm{H}_{5} \mathrm{~N}_{2}\right)_{6}\right]$ is expected to occur at a much lower energy than that at $216 \mathrm{~nm}$ for $\left[\mathrm{Zn}_{4} \mathrm{O}\left(\mathrm{O}_{2} \mathrm{CMe}\right)_{6}\right]$. The broad shoulder at $330 \mathrm{~nm}$ in Fig. 2 may be due to such an electronic transition. For comparison, the analogous $\mathrm{p}_{\pi}\left(\mathrm{O}^{2-}\right) \longrightarrow 4 \mathrm{~s}\left(\mathrm{Zn}^{\mathrm{II}}\right)$ transition of molecular $\mathrm{ZnO}$ occurs at $\lambda<365 \mathrm{~nm}$, depending on the particle size. ${ }^{10}$

The complex exhibits intense photoluminescence both in the solid state and in fluid solutions. Fig. 3 shows the excitation and emission spectra measured in degassed acetonitrile at room temperature. The excitation spectrum is similar to the absorption spectrum. Cooling the solid sample down to $77 \mathrm{~K}$ leads to an enhancement of the emission intensity but without any observable vibronic feature as shown in Fig. 4. In degassed acetonitrile the lifetime and quantum yield of the emission are $0.1 \mu$ s and 0.17 respectively. The long measured lifetime suggests 


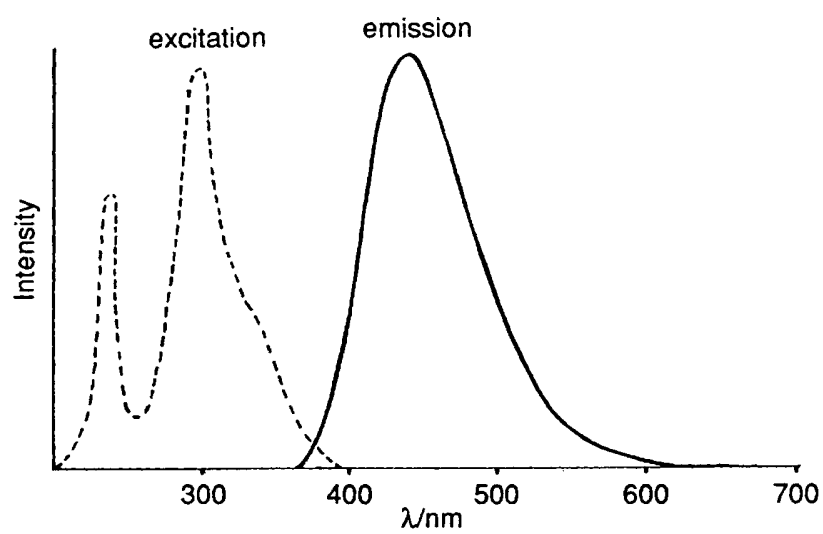

Fig. 3 Excitation and emission spectra of $\left[\mathrm{Zn}_{4} \mathrm{O}\left(\mathrm{C}_{7} \mathrm{H}_{5} \mathrm{~N}_{2}\right)_{6}\right]$ (ca. $\left.10^{-4}-10^{-5} \mathrm{~mol} \mathrm{dm}^{-3}\right)$ in degassed acetonitrile at room temperature

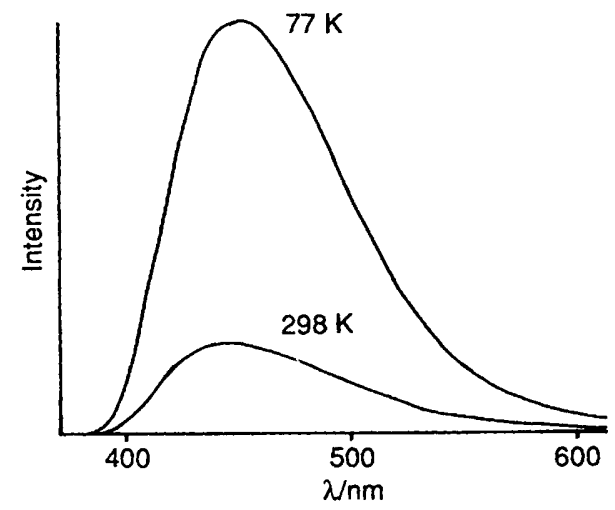

Fig. 4 Emission spectra of a solid sample of $\left[\mathrm{Zn}_{4} \mathrm{O}\left(\mathrm{C}_{7} \mathrm{H}_{5} \mathrm{~N}_{2}\right)_{6}\right]$ at 298 and $77 \mathrm{~K}$

that the emission comes from a spin-forbidden transition. We assign it to the spin-forbidden $4 \mathrm{~S}_{\sigma} \longrightarrow \mathrm{p}_{\pi}\left(\mathrm{O}^{2-}, \mathrm{L}\right)$ transition. Unlike the case of $\left[\{\mathrm{Cu}(\mathrm{py}) \mathrm{I}\}_{4}\right](\mathrm{py}=$ pyridine) where there is a large red shift in emission energy from the solid to solution, ${ }^{3 a}$ the reverse is true for the present complex (solid, 448; acetonitrile, $425 \mathrm{~nm}$ ). This is understandable because in the copper system the emitting electronic excited state is metal-centred and has a stronger metal-metal interaction than in the ground state. In the present zinc(II) system the orbitals involved in the electronic transition do not come from metal-metal bonding interaction which is anticipated to be insignificant for $\mathrm{Zn}^{\text {II }}$.

The $\left[\mathrm{Zn}_{4} \mathrm{O}\left(\mathrm{C}_{7} \mathrm{H}_{5} \mathrm{~N}_{2}\right)_{6}\right]$ complex represents a new zinc(II) cluster having an electronic excited-state lifetime 10 times longer than that for $\left[\mathrm{Zn}_{4} \mathrm{O}\left(\mathrm{O}_{2} \mathrm{CMe}\right)_{6}\right]$. From the low-temperature emission spectrum, the $0-0$ energy of the long-lived excited-state molecule is estimated to be around $400 \mathrm{~nm}(3.2 \mathrm{eV}, c a$. $\left.5 \times 10^{-19} \mathrm{~J}\right)$. Such a high energy and long-lived excited-state molecule is uncommon in the chemistry of luminescent metal clusters and may be a potential sensitizer for photochemical energy-transfer processes.

\section{Acknowledgements}

We acknowledge support from the National Science Council of Taiwan. C.-M. C. thanks the Croucher Foundation and the Hong Kong Research Grants Council for a visiting professorship, administered by the National Taiwan University.

\section{References}

1 P. D. Harvey and H. B. Gray, J. Am. Chem. Soc., 1988, 110, 2145; P. D. Harvey, F. Adar and H. B. Gray, J. Am. Chem. Soc., 1989, 111, 1312.

2 C. M. Che, W. T. Wong, T. F. Lai and H. L. Kwang, J. Chem. Soc., Chem Commun., 1989, 243: C. M. Che, H. L. Kwang, C. K. Poon and V. W. W. Yam, J. Chem. Soc., Dalton Trans., 1990, 3215; V. W. W. Yam, T. F. Lai and C. M. Che, J. Chem. Soc., Dalton Trans., 1990, 3747; C. M. Che, H. K. Yip, V. W. W. Yam, P. Y. Cheung, T. F. Lai, S. J. Shieh and S. M. Peng, J. Chem. Soc., Dalton Trans., 1992, 427.

3 (a) K. R. Kyle, C. K. Ryu, J. A. DiBenedetto and P. C. Ford, J. Am. Chem. Soc., 1991, 113, 2954; (b) M. Henary and J. I. Zink, J. Am. Chem. Soc., 1989, 111, 7407.

4 (a) A. Vogler and H. Kunkely, J. Am. Chem. Soc., 1986, 108, 7211; (b) A. Vogler and H. Kunkely, Chem. Phys. Lett., 1988, 150, 135; (c) A Vogler and H. Kunkely, Chem. Phys. Lett., 1989, 158, 74; (d) A. Vogler and H. Kunkely, J. Chem. Soc., Chem. Commun., 1990, 1204; (e) T. Turk, U. Resch, M. A. Fox and A. Vogler, Inorg. Chem., 1992, 31, 1854.

5 R. Bertoncello, M. Bettinelli, M. Casarin, A. Gulino, E. Tondello and A. Vittadini, Inorg. Chem., 1992, 31, 1558.

6 E. J. Gabe, Y. Le Page, J.-P. Charland, F. L. Lee and P. S. White, J. Appl. Crystallogr., 1989, 22, 384.

7 J. N. Demas and G. A. Crosby, J. Phys. Chem., 1971, 75, 991.

8 S. M. Peng and Y. N. Lin, Acta Crystallogr., Sect. C, 1986, 42, 1725.

9 H. Koyama and Y. Saito, Bull. Chem. Soc. Jpn., 1954, 27, 112.

10 D. W. Bahnemann, C. Kormann and M. R. Hoffmann, J. Phys. Chem., 1987, 91, 3789.

Received 15th July 1992; Paper 2/03770J 